\title{
Perfil de suscetibilidade de leveduras do gênero Candida isoladas de animais ao óleo essencial de Rosmarinus officinalis $\mathrm{L}$.
}

\author{
CLEFF, M.B. ${ }^{1 *}$; MEINERZ, A.R.M. ${ }^{1}$; MADRID, I. ${ }^{2}$; FONSECA, A.O. ${ }^{2}$; ALVES, G.H. ${ }^{3}$; MEIRELES, M.C.A. ${ }^{2}$; \\ RODRIGUES, M.R.A. ${ }^{3}$ \\ ${ }^{1}$ Departamento de Clínicas Veterinária, ${ }^{2}$ Departamento de Veterinária Preventiva, Faculdade de Veterinária, \\ Universidade Federal de Pelotas, Campus Universitário, CEP: 96010-900, Pelotas-Brasil *emebrum@bol.com.br \\ ${ }^{3}$ Instituto de Química e Geociências, UFPel. Campus Universitário. CEP: 96010-900, Pelotas-Brasil
}

\begin{abstract}
RESUMO: Neste trabalho foram avaliadas a atividade antifúngica e composição química do óleo essencial de Rosmarinus officinalis (alecrim). O óleo essencial foi obtido, através da hidrodestilação, das partes aéreas da planta, o qual foi analisado por cromatografia gasosa com detectores de ionização em chama (GC/FID) e espectrometria de massas (GC/MS). Esse óleo foi testado em isolados de $C$. albicans da mucosa vaginal de fêmeas caninas, casos clínicos de candidíase em animais cepas padrões e espécies não-albicans, usando a técnica de microdiluição em caldo (CLSI-M27A2). A análise cromatográfica do óleo permitiu identificar como principais compostos cânfora, verbenona e 1,8-cineol. Os valores de CIM do óleo para os isolados padrões de $C$. albicans variaram entre 1,25 e 2,5 $\mu \mathrm{L} \mathrm{mL}^{-1}$ e de 2,5 a $5,0 \mu \mathrm{L} \mathrm{mL}^{-1}$ para CFM, enquanto que para os isolados não-albicans observou-se CIM entre 1,25 e 5,0 $\mu \mathrm{L} \mathrm{mL}^{-1}$ e CFM de 2,5 a 10,0 $\mu \mathrm{L} \mathrm{mL}^{-1}$. C. albicans isolada de animais apresentou valores de $2,5 \mathrm{a} \geq 10,0 \mu \mathrm{L} \mathrm{mL}^{-1}$ para CIM e de 5,0 a $\geq 10,0 \mu \mathrm{L} \mathrm{mL}-1$ para CFM. Os resultados demonstraram atividade fungicida e fungistática do óleo essencial de alecrim em isolados de Candida spp. provenientes de animais.
\end{abstract}

Palavras-chave: alecrim, óleo essencial, Candida spp., animais

\begin{abstract}
Susceptibility profile of Candida spp. isolated from animals to the essential oil of Rosmarinus officinalis L. In this work the antifungal activity and chemical composition of the Rosmarinus officinalis (rosemary) essential oil against Candida spp. were evaluated. The essential oil was obtained by hydrodistillation of aerial parts, and analyzed by gas chromatography with a flame ionization detector (GC/FID) and gas chromatography coupled with mass spectrometry (GC/MS). This oil was tested against fourteen isolates of Candida sp. (ten C. albicans and four no-albicans species) by the microdillution broth assay (CLSI-M27A2). Chromatography analyses of the essential oil showed compounds, as camphor, verbenone and 1,8-cineole as major constituents. MIC and MFC values for $C$. albicans standard strains were $1.25-2.5 \mu \mathrm{L} \mathrm{mL}^{-1}$ and $2.5-5.0 \mu \mathrm{L} \mathrm{mL}-1$, respectively. MIC and MFC for the non-albicans species were $1.25-5.0 \mu \mathrm{L} \mathrm{mL}^{-1}$ and $2.5-10.0 \mu \mathrm{LL}^{-1}$, respectively. C. albicans isolates obtained from animals exhibited MIC and MFC values of $2.5-\geq 10.0 \mu \mathrm{L} \mathrm{mL}^{-1}$ and $5.0-\geq 10.0 \mu \mathrm{L} \mathrm{mL}^{-1}$, respectively. According to the results, the essential oil of rosemary presented fungicidal and fungistatic activities against Candida spp.
\end{abstract}

Key words: rosemary, essential oil, Candida spp, animals

\section{INTRODUÇÃO}

Candida spp. são leveduras comensais do trato gastrointestinal, respiratório e membranas mucosas de animais domésticos (Cleff et al., 2005; Moretti et al., 2006; Yurayart et al., 2011). C. albicans destaca-se como principal agente etiológico nos casos de candidíase em animais de companhia, porém outras espécies como C. parapsilosis, C. guillermondii e C. tropicalis podem estar envolvidas em diferentes processos patogênicos (Moretti et al., 2006; Cleff et al., 2007; Pozzatti, 2007; Yurayart et al., 2011). A infecção oportunista em animais desenvolve-se como resultado da quebra dos mecanismos de defesa inata, imunossupressão e antibioticoterapia prolongada, entre outros fatores

Recebido para publicação em 06/12/2009

Aceito para publicação em 11/09/2011

Rev. Bras. PI. Med., Botucatu, v.14, n.1, p.43-49, 2012. 
(Moretti et al., 2006; Cleff et al., 2007; Ong et al., 2010; Yurayart et al., 2011). Atualmente, candidíase representa um problema de saúde pública, com resistência de isolados aos antifúngicos convencionais, e relatos de recidivas da enfermidade em especial nos pacientes imunocomprometidos (Sanglard \& Odds, 2002; Colombo \& Guimarães, 2003; Pozzatti et al., 2010).

Estudos com extratos vegetais visam buscar compostos ativos com amplo espectro de ação, baixa toxicidade e custo reduzido (Rochete et al., 2003; Angioni et al., 2004; Bernardes et al., 2010), uma vez que os antifúngicos disponíveis para o tratamento de micoses em medicina veterinária é limitado, restringindo-se aos polienos convencionais e triazóis. Além disso, estes fármacos apresentam efeitos adversos e custo relativamente elevado, especialmente quando considerado o uso em animais (Sanglard \& Odds, 2002; Mondelo et al., 2003; Pressler et al., 2003; Rochete et al., 2003; Giordani et al., 2004; Luqman et al., 2007).

Dentre as plantas da família Lamiaceae, o alecrim (Rosmarinus officinalis L.) é amplamente utilizado como tônico ou condimento culinário assim como, na forma de chás para o tratamento de problemas digestivos (Simões \& Spitzer, 2003). A planta se apresenta como arbusto aromático de pequeno porte, capaz de produzir óleos essenciais, que são uma mistura complexa de compostos, oriundos do metabolismo secundário (Simões \& Spitzer, 2003; Gobbo-Neto \& Lopes, 2006; Gachkar et al., 2007). No óleo essencial de alecrim, destacamse como principais constituintes os terpenos como cânfora, 1,8-cineol, $\alpha$-pineno, verbenona, borneol e piperitona, os quais são reconhecidos por apresentar atividades inseticidas, antioxidantes e antimicrobianas (Angioni et al., 2004; Kabouche et al., 2005; Sacchetti et al., 2005; Santoyo et al., 2005; Prins et al., 2006; Celiktas et al., 2007; Gachkar et al., 2007). Embora se reconheça a capacidade antimicrobiana de óleos de várias plantas, o mecanismo de ação ainda é desconhecido, sendo que algumas teorias tem sido propostas (Sikkema et al., 1995; Cox et al., 2000; Lambert et al., 2001; Koschier et al., 2002; Sartoratto et al., 2004).

A ação antimicrobiana do alecrim, tanto na forma de extratos como de óleo essencial, tem sido avaliada principalmente em bactérias dos gêneros Staphylococcus, Streptococcus, Proteus, Pseudomonas, Klebsiella, Salmonella, Mycobacterium e Escherichia (Porte \& Godoy, 2001; Angioni et al., 2004; Santoyo et al., 2005; Celiktas et al., 2007; Luqman et al., 2007; Bernardes et al., 2010; Hussain et al., 2010). Nas leveduras do gênero Candida, os estudos concentram-se em isolados provenientes de humanos e cepas padrões (Sacchetti et al., 2005; Lima et al., 2006; Pinto et al., 2006;
Schwiertz et al., 2006; Celiktas et al., 2007; Luqman et al., 2007; Pozzatti et al., 2010; Cavalcanti et al., 2011) não sendo encontradas na literatura consultada, pesquisas que avaliem o efeito do óleo essencial do alecrim em $C$. albicans isolada de mucosa e casos clínicos em animais de companhia e silvestres. Nesse sentido, o presente estudo teve como objetivos identificar os principais constituintes químicos, usando a cromatografia gasosa no óleo essencial de alecrim cultivado em Pelotas/RS e, avaliar as propriedades antifúngicas do óleo essencial em Candida albicans isoladas da microbiota e de casos clínicos de candidíase em animais, assim como em isolados padrões de Candida spp.

\section{MATERIAL E MÉTODO}

\section{Material Vegetal}

As partes aéreas do alecrim (Rosmarinus officinalis L.) foram colhidas no mês de fevereiro de 2008, na cidade de Pelotas/RS/Brasil, período em que a planta estava com grande quantidade de material vegetal. Após a identificação botânica, a exsicata foi depositada no herbário da Universidade Federal de Pelotas - UFPel, e registrada sob no PEL 24.600. O material coletado foi seco em estufa com circulação de ar (Marconi MA 035) em temperatura de $40^{\circ} \mathrm{C}$. As folhas e flores secas foram acondicionadas em frascos hermeticamente fechados, sendo que antes do processo de obtenção do óleo, foi determinada a umidade do material com base na metodologia descrita na Farmacopéia Brasileira IV (1988).

\section{Extração do Óleo Essencial}

Cerca de $100 \mathrm{~g}$ do material vegetal seco foram submetidos à extração por hidrodestilação, em aparelho tipo Clevenger, durante quatro horas, em triplicata. O óleo essencial obtido foi seco em Na $\mathrm{SO}_{4}$ (anidro, grau p.a, Merck) e concentrado sob $\mathrm{N}_{2}$ (ultra puro, 99,99\%, White Martins), armazenado em frasco âmbar sob refrigeração até a utilização, tanto nas análises cromatográficas, como na determinação da atividade antifúngica (Farmacopéia Brasileira IV, 1988; Rodrigues et al., 2004).

\section{Análise Cromatográfica do Óleo Essencial de alecrim \\ O óleo essencial foi analisado por} cromatografia gasosa em dois equipamentos (GC/ FID e GC/MS), visando à identificação dos constituintes, por comparação com o tempo de retenção de padrões cromatográficos e, confirmação pelos espectros de massas constantes na biblioteca do equipamento.

A análise cromatográfica, através do equipamento GC/FID (Schimadzu-17 A) equipado com

Rev. Bras. PI. Med., Botucatu, v.14, n.1, p.43-49, 2012. 
uma coluna de sílica DB-5 (30 m x 0,25 mm x 0,25 $\mu \mathrm{m})$ foi realizada nas seguintes condições: aquecimento a $40^{\circ} \mathrm{C}$, mantendo a $2^{\circ} \mathrm{C} \mathrm{min}^{-1}$ até $145^{\circ} \mathrm{C}$, depois a $10^{\circ} \mathrm{C} \mathrm{min}-1$ até $280^{\circ} \mathrm{C}$, permanecendo por 10 $\min ; \mathrm{T}_{\text {detector }}=\mathrm{T}_{\text {injetor }}=280^{\circ} \mathrm{C} ; \mathrm{T}_{\text {coluna }}=40^{\circ} \mathrm{C}$; razão de split de 1:50. Foram preparadas soluções em hexano $\left(5.000 \mu \mathrm{g} \mathrm{mL}^{-1}\right)$ dos óleos essenciais, das quais foram retiradas alíquotas de $1 \mu \mathrm{L}$ para injeção no cromatógrafo. Também foi preparada uma solução (40 $\mu \mathrm{g} \mathrm{mL}^{-1}$ ) dos padrões cromatográficos ( $\alpha$-pineno, canfeno, $\beta$-pineno, mirceno, $\alpha$-terpineno, $p$-cimeno, limoneno, 1,8-cineol, $\alpha$-terpineno, terpinoleno, linalol, 4-terpineol, $\alpha$-terpineol, timol e carvacrol), que foi submetida às mesmas condições das amostras. Os compostos nas amostras foram identificados por comparação com o tempo de retenção dos padrões terpênicos e com dados da literatura (Rodrigues et al., 2004).

A análise cromatográfica através do equipamento GC/MS (Schimadzu-5050 A) equipado com uma coluna de sílica OV-05 (60 m x 0,25 mm x $0,25 \mu \mathrm{m})$ foi realizada nas seguintes condições: aquecimento a $60^{\circ} \mathrm{C}$, mantendo a $3^{\circ} \mathrm{C} \mathrm{min}{ }^{-1}$ até $250^{\circ} \mathrm{C}$, depois a $10^{\circ} \mathrm{C} \mathrm{min}-1$ até $280^{\circ} \mathrm{C}$, permanecendo por 10 $\min ; \mathrm{T}_{\text {interface }}=\mathrm{T}_{\text {injetor }}=250^{\circ} \mathrm{C} ; \mathrm{T}_{\text {coluna }}=60^{\circ} \mathrm{C}$; razão de split de 1:10; $E=2,0 \mathrm{KeV}$; fluxo $=1,0 \mathrm{~mL} \mathrm{~m}^{-1}$; modo = SCAN. Foram usadas as mesmas soluções dos óleos e dos padrões das análises no GC/FID, sendo injetados $1 \mu \mathrm{L}$ de cada solução, na qual os constituintes foram identificados por comparação com os espectros de massa da biblioteca Wiley do equipamento e por comparação com tempo de retenção dos padrões utilizados (Rodrigues et al., 2004).

\section{Obtenção dos isolados fúngicos}

Para a realização do estudo foram utilizados 14 isolados de Candida spp. Seis isolados padrões foram cedidos pela Fundação Oswaldo Cruz (Fiocruz/ Rio de Janeiro/Brasil), tais como: C. albicans (ATCC44858 e 18804), C. parapsilosis (ATCC 22019), C. krusei (ATCC 34135), C. Iusitaniae (ATCC 34449) e C. dubliniensis (ATCC MY646). Os outros utilizados foram oito isolados de campo, pertencentes à espécie C. albicans, sendo seis provenientes da mucosa vaginal de fêmeas caninas e dois de casos clínicos de candidíase cutânea em cão ( $n=1$ ) e macaco-prego (Cebus apella) ( $n=1)$, os quais estavam estocados no Setor de Micologia do Laboratório de Doenças Infecciosas da Faculdade de Veterinária da UFPel.

\section{Teste in vitro da atividade inibitória}

A suscetibilidade fúngica ao óleo essencial de alecrim foi avaliada através da técnica de micro diluição em caldo (CLSI-M27-A2) adaptada para um fitofármaco, com a adição de $0,1 \%$ de Tween 80 ao meio RPMI 1640 como dispersante do óleo essencial, a fim de inibir a formação de micelas.

O inóculo de Candida spp foi preparado em solução salina estéril a partir de colônias jovens da levedura com $24 \mathrm{~h}$ de crescimento, sendo ajustado na turbidez da escala $1 / 2$ de McFarland correspondendo a 1-5 x 106 UFC $\mathrm{mL}^{-1}$, sendo posteriormente diluído em solução salina estéril (1:100) e em meio RPMI 1640 (1:20), obtendo-se concentração final de 1-5 x $10^{3} \mathrm{UFC} \mathrm{mL} \mathrm{mL}^{-1}$.

Para a avaliação da CIM (Concentração Inibitória Mínima) do óleo essencial de alecrim frente à Candida spp, foram utilizadas 10 diluições sucessivas em meio RPMI, obtendo-se concentrações de 10 a $0,02 \mu \mathrm{L} \mathrm{mL}^{-1}$ as quais foram dispostas num volume de $100 \mu \mathrm{L}$ no sentido das colunas da placa de microdiluição. Os testes foram realizados em duplicata, sendo utilizada uma coluna como controle positivo (inóculo/meio) e uma coluna como controle negativo (óleo essencial/meio). As microplacas foram incubadas a $35^{\circ} \mathrm{C}$ por $48 \mathrm{~h}$ e a interpretação dos resultados foi realizada através da visualização da turvação referente à multiplicação do microrganismo, comparada aos controles positivo e negativo. Dessa forma, a CIM foi definida como a menor concentração capaz de produzir inibição do crescimento de Candida spp. em relação ao controle positivo. Para determinação da concentração fungicida mínima (CFM), uma alíquota de $10 \mu \mathrm{L}$ de cada poço da microplaca foi semeada em placas contendo ágar Sabouraud dextrose, incubadas por $24 \mathrm{~h}$ para observação do crescimento das leveduras.

\section{Análise estatística}

Os resultados de CIM e CFM entre os diferentes isolados de Candida spp foram comparados através de análise de variância e a comparação entre as médias geométricas foi realizada de acordo com teste de Tukey, utilizando o programa estatístico Statistix 6.0, sendo considerado significativo quando $p \leq 0,05$.

\section{RESULTADO E DISCUSSÃO}

A avaliação botânica do material vegetal, utilizado no estudo, confirmou a espécie como Rosmarinus officinalis L., a qual apresentou rendimento em óleo de $3,24 \%(\mathrm{v} / \mathrm{m})$. Os resultados foram superiores aos observados por Prins et al. (2006) que obtiveram rendimento de $2 \%$ para o óleo de $R$. officinalis e por Porte \& Godoy (2001) que descreveram rendimento de 0,5 a 2,5\% de óleo.

$\mathrm{Na}$ análise cromatográfica do óleo essencial do $R$. officinalis foi evidenciada a presença de 22 compostos (Tabela 1), sendo que os principais constituintes identificados, usando padrões cromatográficos, foram a cânfora $(\sim 56,00 \%), 1,8$ cineol $(\sim 16,00 \%)$, verbenona $(\sim 7,80 \%)$ e mirceno 
( 4,00\%), via GC/FID e confirmados pelos espectros de massas do GC/MS. O uso das duas técnicas permitiu a identificação e confirmação dos constituintes químicos do óleo essencial de alecrim.

Os constituintes químicos encontrados no óleo estudado estão de acordo com os resultados obtidos por outros autores, apenas com pequenas variações (Giordani et al., 2004; Kabouche et al., 2005; Celiktas et al., 2007; Gachkar et al., 2007). Alguns trabalhos demonstraram a presença de verbenona e borneol constituindo aproximadamente $80 \%$ do óleo essencial (Santoyo et al., 2005), enquanto outros citaram o 1,8-cineol (Kabouche et al., 2005); borneol, canfeno, verbenona e $\alpha$-pineno (Angioni et al., 2004), ou ainda, cânfora, eucaliptol e $\beta$-mirceno (Prins et al., 2006) como principais constituintes. Hussain et al. (2010) ao analisarem via GC/MS o óleo essencial de $R$. officinalis proveniente do Paquistão, encontraram 18 componentes químicos, representados principalmente por 1,8 cineol $(38,5 \%)$, cânfora $(17,1 \%)$ e $\alpha$-pineno (12,3\%). As diferenças na composição do óleo de alecrim têm sido atribuídas principalmente a efeitos climáticos e/ou época do ano, altitude, formas de cultivo, condições de secagem e estocagem, formas de extração, incluindo velocidade de aquecimento e o tempo de extração, entre outros, o que influencia na composição, atividade, qualidade e quantidade de óleo essencial presente nos vegetais (Gobbo-Neto \& Lopes, 2006; Prins et al., 2006; Celiktas et al., 2007).

A potencialidade antimicrobiana do alecrim tem sido demonstrada, utilizando-se técnicas adaptadas de antifungigrama como, difusão em ágar, difusão em disco e diluição em caldo (Lambert et al., 2001; Angioni et al., 2004; Santoyo et al., 2005; Schwiertz et al., 2006; Gachkar et al., 2007). Nesse estudo, a atividade antifúngica do óleo foi determinada através de microdiluição em caldo, sendo a CIM e CFM considerados os melhores parâmetros para avaliar a ação antifúngica de óleos essenciais (Pozzatti, 2007).

Os resultados demonstraram diferenças de suscetibilidade entre isolados de campo e cepas padrões, assim como entre as espécies de Candida ao óleo essencial de alecrim, não sendo estatisticamente significativa. Os isolados de $C$. albicans, obtidos da mucosa dos animais foram sensíveis em concentrações médias de $5,0 \mu \mathrm{L} \mathrm{mL}^{-1}$, enquanto que os dois isolados de casos clínicos não apresentaram sensibilidade (Tabela 2), sendo necessárias outras avaliações, utilizando maiores concentrações do óleo nestes isolados, a fim de esclarecer esta observação. Cabe salientar, que no caso do isolado de tegumento cutâneo de cão, o animal havia sido tratado com cetoconazol tópico e oral por 30 dias, sem a resolução das lesões. Quanto ao isolado fúngico proveniente de macaco-prego, este foi obtido sem haver o tratamento prévio do animal com antifúngicos.

A atividade anticandida do óleo essencial de alecrim tem sido avaliada por outros autores resultando em amplos valores de inibição (Giordani et al., 2004; Sachetti et al., 2005; Santoyo et al., 2005; Celiktas et al., 2007), os quais são apresentados em diferentes unidades, o que dificulta as comparações entre os trabalhos. Entretanto, esses

TABELA 1. Compostos identificados no óleo essencial de alecrim, utilizando as técnicas cromatográficas GC/FID e GC/MS.

\begin{tabular}{|c|c|c|c|c|c|}
\hline № & Compostos C & $(\%)^{* *}$ & № & Compostos C & $(\%)^{* *}$ \\
\hline 1 & $\alpha$-pineno* & 0,47 & 12 & Carenal & 0,44 \\
\hline 2 & Canfeno* & 0,29 & 13 & Cânfora & 56,04 \\
\hline 3 & â-pineno* & 0,21 & 14 & cis ou trans-hidrocarveol & 0,40 \\
\hline 4 & mirceno* & 4,06 & 15 & Pinocarvona & 0,23 \\
\hline 5 & $\alpha$-terpineno* & 0,29 & 16 & Borneol & 2,03 \\
\hline 6 & $p$-cimeno* & 1,53 & 17 & cis ou trans-hidrocarveol & 0,45 \\
\hline 7 & limoneno* & 0,46 & 18 & Isopinocanfona & 0,55 \\
\hline 8 & 1,8 -cineol* & 16,02 & 19 & 4-terpineol* & 1,39 \\
\hline 9 & $\gamma$-terpineno* & 0,55 & 20 & $\alpha$-terpineol* & 3,74 \\
\hline 10 & terpinoleno* & 0,43 & 21 & Verbenona & 7,76 \\
\hline 11 & linalol* & 2,03 & 22 & $\beta$-cariofileno & 0,63 \\
\hline
\end{tabular}

${ }^{*}$ Compostos identificados por comparação com padrões, via GC/FID e GC/MS; demais compostos identificados por comparação com espectros de massa da biblioteca Wiley do equipamento GC/MS; ${ }^{* *}$ Concentração em percentual, calculada em relação as áreas normalizadas dos picos

Rev. Bras. Pl. Med., Botucatu, v.14, n.1, p.43-49, 2012. 
TABELA 2. Valores de CIM e CFM do óleo essencial do alecrim em leveduras do gênero Candida isoladas de animais e cepas padrões

\begin{tabular}{llcc}
\hline Origem & Isolados & ClM $^{*} \mu \mathbf{L ~ m L}^{-1}$ & CFM $^{* *} \mu \mathbf{~ m L}^{-1}$ \\
\hline Mucosa vaginal de fêmeas caninas & C. albicans & 5,0 & 5,0 \\
& C. albicans & 2,5 & 5,0 \\
& C. albicans & 5,0 & 5,0 \\
& C. albicans & 10,0 & 10,0 \\
& C. albicans & 5,0 & 5,0 \\
& C. albicans & 2,5 & 5,0 \\
\hline Casos clínicos de candidíase & C. albicans & $\geq 10,0$ \\
& C. albicans & \\
& C. albicans (ATCC 44858) & 1,25 & $\geq 10,0$ \\
\hline Cepas padrões & C. albicans (ATCC 18804) & 2,5 & 5,0 \\
& C. dubliniensis (MY 646) & 5,0 & 2,5 \\
& C. parapsilosis (ATCC 22019) & 1,25 & 10,0 \\
& C. lusitanie (ATCC 34449) & 1,25 & 5,0 \\
& C. krusei (ATCC 34135) & 5,0 & 2,5 \\
\hline
\end{tabular}

${ }^{*} \mathrm{CIM}$ : concentração inibitória mínima; ${ }^{* *}$ CFM: Concentração fungicida mínima; ${ }^{* *}$ Tegumento cutâneo de cão; ${ }^{* * *}$ Tegumento cutâneo de macaco-prego.

resultados estão de acordo com Cavalcanti et al. (2011) que demonstraram CIM e CFM igual a 2,5 $\mu \mathrm{L}$ $\mathrm{mL}^{-1}$ para o óleo de alecrim em isolados padrões de C. albicans, e com Giordani et al. (2004) que demonstrou CIM de 5,6 $\mu \mathrm{L} \mathrm{mL}^{-1}$ para C. albicans. Em contrapartida, estudo realizado com $C$. albicans, C. dubliniensis, C. glabrata, C. krusei e C. tropicalis obtidos de humanos e cepas padrões, demonstrou resistência dos isolados nas concentrações de $50 \mu \mathrm{L}$ $\mathrm{mL}^{-1}$ a $3200 \mu \mathrm{L} \mathrm{mL}^{-1}$ de óleo essencial de alecrim, cujos componentes majoritários eram 1,8-cineol e cânfora (Pozzatti, 2007).

Com relação às cepas de referência, as espécies C. lusitane, C. parapsilosis e C. albicans foram as mais sensíveis ao óleo essencial, enquanto C. dublinensis e C. krusei apresentaram maiores valores de CIM e CFM. Com relação a C. krusei, sugere-se que características na estrutura celular, assim como exacerbada ação das bombas de efluxo, dificultariam a entrada dos óleos essenciais na célula, podendo assim apresentar-se mais resistente (Mondello et al., 2003; Pozzatti, 2007).

Diferenças na atividade do óleo de alecrim têm sido relacionadas com a localização geográfica da planta e variação sazonal (Celiktas et al., 2007). Estudos demonstraram maior atividade antimicrobiana de plantas colhidas na primavera e que apresentou como principal constituinte o borneol, seguido pela cânfora e verbenona (Koschier et al.,
2002; Sacchetti et al., 2005). Além disso, a associação de componentes também influencia no potencial antimicrobiano dos óleos essenciais (Koschier et al., 2002; Sachetti et al., 2005; Gachkar et al., 2007).

Apesar do reconhecimento das propriedades antimicrobianas, o mecanismo de ação dos extratos e/ou óleos essenciais ainda não está completamente elucidado, porém estudos têm sido conduzidos neste sentido (Sikkema et al., 1995; Cox et al., 2000; Lambert et al., 2001; Sartoratto et al., 2004; Pinto et al., 2006). Segundo Lambert et al (2001), a ação antimicrobiana pode ser devido ao prejuízo à diferentes enzimas, principalmente aquelas envolvidas com a produção de energia e/ou síntese de componentes estruturais dos microrganismos. Outra hipótese seria de que os óleos essenciais sensibilizam a bicamada fosfolipídica da membrana celular, causando um aumento da permeabilidade e perdas de constituintes intracelulares vitais (Cox et al., 2000), ou ainda a ocorrência de danos na membrana citoplasmática e redução no conteúdo de ergosterol dos fungos (Pinto et al., 2006).

De acordo com estes resultados foi possível concluir que o óleo essencial de alecrim, contendo cânfora, 1,8-cineol e verbenona como principais constituintes, apresentou atividade fungicida e fungistática in vitro em leveduras do gênero Candida. A importância destes resultados é evidenciada pela 
problemática da terapêutica antifúngica, quando se refere a Candida spp., as quais tem desenvolvido resistência aos azólicos, que são os fármacos de eleição para a candidíase em animais.

\section{AGRADECIMENTO}

À Dra Élen Nunes Garcia pela identificação botânica do alecrim utilizado no presente estudo, CNPq, FAPERGS e CAPES.

\section{REFERÊNCIA}

ANGIONI, A. et al. Chemical composition, plant genetic differences, antimicrobial and antifungal activity investigation of the essential oil of Rosmarinus officinalis L. Journal of Agricultural and Food Chemistry, v.52, n.11, p.3530-5, 2004.

BERNARDES, W.A. et al. Antimicrobial activity of Rosmarinus officinalis against oral pathogens: relevance of carnosic acid and carnosol. Chemistry \& Biodiversity, v.7, n.7, p.1835-40, 2010.

CAVALCANTI, Y.W.; ALMEIDA, L.F.D.; PADILHA, W.W.N. Antifungal activity of tinctures from natural products on Candida spp. International Journal of Dentistry, v.10, n.1, p.15-9, 2011.

CELIKTAS, O.Y. et al. Antimicrobial activities of methanol extracts and essential oils of Rosmarinus officinalis, depending on location and seasonal variations. Food Chemistry, v.1, n.100, p.553-9, 2007.

CLEFF, M.B. et al. Isolation of Candida spp from vaginal microbiota of healthy canine females during estrous cycle. Brazilian Journal of Microbiology, v.36, n.2, p.2014, 2005.

CLEFF, M.B. et al. Infecção cutânea em cão por Candida albicans. Revista Veterinária e Zootecnia, v.14, n.2, p.164-8, 2007.

CLSI (Clinical and Laboratory Standards Institute). Método de referência para testes de diluição em caldo para determinação da sensibilidade de leveduras à terapia antifúngica: norma aprovada - M27-A2. 2.ed. Wayne,2002.

COLOMBO, A.L.; GUIMARÃES, T. Epidemiologia das infecções hematogênicas por Candida spp. Revista da Sociedade Brasileira de Medicina Tropical, v.36, n.5, p.599-607, 2003.

COX, S.D. et al. The mode of antimicrobial action of the essential oil of Melaleuca alternifolia (tea tree oil). Journal Applied of Microbiology, v.88, n.1, p.170-5, 2000. Farmacopéia Brasileira. 4.ed. São Paulo: Atheneu, Parte I, 1988. Paginação irregular.

GACHKAR, L. et al. Chemical and biological characteristics of Cuminum cyminum and Rosmarinus officinalis essential oils. Food Chemistry, v.102, n.3, p.898-904, 2007.

GIORDANI, R. et al. Antifungal effect of various essential oils against Candida albicans. Potentiation of antifungal action of amphotericin B by essential oil from Thymus vulgaris. Phytotherapy Reserch, v. 18, n.12, p.990-5, 2004. GOBBO-NETO, L.; LOPES, N.P. Plantas medicinais: fatores de influência no conteúdo de metabólitos secundários. Química Nova, v.30, n.2, p.374-81, 2006. HUSSAIN, A.I. et al. Rosmarinus officinalis essential oil: antiproliferative, antioxidant and antibacterial activities. Brazilian Journal of Microbiology, v.41, n.4, p.1070-8, 2010.

$\mathrm{KABOUCHE}$, Z. et al. Comparative antibacterial activity of five Lamiaceae essential oils from Algeria. International Journal Aromatherapy, v.15, n.3, p.129-33, 2005.

KOSCHIER, E.H.; SEDY, K.A.; NOVAK, J. Influence of plant volatiles on feeding damage caused by the onion thrips Thrips tabaci. Crop Protection, v.21, n.5, p.419-25, 2002. LAMBERT, R.J.W.; SKANDAMIS, P.N.; COOTE, P.J. Astudy of the minimum inhibitory concentration and mode of action of oregano essential oil, thymol and carvacrol. Journal Applied of Microbiology, v.91, n.3, p.453-62, 2001.

LIMA, I.O. et al. Atividade antifúngica de óleos essenciais sobre espécies de Candida. Revista Brasileira de Farmacognosia, v.16, n.2, p.197-201, 2006.

LUQMAN, S. et al. Potential of rosemary oil to be used in drug-resistant infections. Alternative Therapies in Health and Medicine, v.13, n.5, p.54-9, 2007.

MONDELO, F. et al. In vitro and in vivo activity of tea tree oil against azole-susceptible and resistant human patogenic yeasts. Journal of Antimicrobial Chemotherapy, v.51, n.5, p.1223-9, 2003.

MORETTI, A. et al. Co-cutaneous infection in a dog: pcrreverse identification of $C$. tropicalis on skin biopsy. Journal of Mycologie Medicale, v.16, n.1, p.30-6, 2006. ONG, R.K.; RAISIS, A.L.; SWINDELLS, K.L. Candida albicans peritonitis in a dog. Journal of Veterinary Emergency and Critical Care, v.20, n.1, p.143-7, 2010.

PINTO, E. et al. Antifungal activity of the essential oil of Thymus pulegioides on Candida, Aspergillus and dermatophyte species. Journal of Medical Microbiology, v.55, n.10, p.1367-73, 2006.

PORTE, A.; GODOY, R.L.O. Alecrim (Rosmarinus officinalis L.): propriedades antimicrobianas e químicas do óleo essencial. Boletim Centro de Pesquisa de Processamento de Alimentos, v.19, n.2, p.193-210, 2001. POZZATTI, P. Suscetibilidade de Candida spp sensíveis e resistentes ao fluconazol frente a óleos essenciais extraídos de condimentos. 2007. 148p. Dissertação (Mestrado - Área de Concentração em Ciências Farmacêuticas) - Centro de Ciências da Saúde, Universidade Federal de Santa Maria, Santa Maria.

POZZATTI, P. et al. Comparison of the susceptibilities of clinical isolates of Candida albicans and Candida dubliniensis to essential oils. Mycoses, v.53, n.1, p.125, 2010.

PRESSLER, B.M. et al. Candida spp. urinary tract infections in 13 dogs and seven cats: predisposing factors, treatment, and outcome. Journal of the American Animal Hospital Association, v.39, n.3, p.26370, 2003.

PRINS, C.L.; LEMOS, C.L.S.; FREITAS, S.P. Efeito do tempo de extração sobre a composição e o rendimento do óleo essencial de alecrim (Rosmarinus officinalis). Revista Brasileira de Plantas Medicinais, v.8, n.4, p.925, 2006.

ROCHETE, F.; ENGELEN, M.; VANDEN BOSSCHE, H. Antifungal agents of use in animal health-pratical 
applications. Journal Veterinary Pharmacology and Therapy, v.26, n.1, p.31-53, 2003.

RODRIGUES, M.R.A. et al. Chemical composition and extraction yield of the extract of Origanum vulgare obtained from sub- and supercritical $\mathrm{CO}_{2}$. Journal of Agricultural and Food Chemistry, v.52, n.10, p.3042-7, 2004.

SACCHETTI, G. et al. Comparative evaluation of 11 essential oils of different origin as functional antioxidants, antiradicals and antimicrobials in foods. Food Chemistry, v.91, n.4, p.621-32, 2005.

SANGLARD, D.; ODDS, F.C. Resistance of Candida species to antifungal agents: molecular mechanisms and clinical consequences. The Lancet Infections Diseases, v.2, n.2, p.73-85, 2002.

SANTOYO, S. et al. Chemical composition and antimicrobial activity of Rosmarinus officinalis L. essential oil obtained via supercritical fluid extraction. Journal of Food Protection, v.68, n.4, p.790-5, 2005.
SARTORATTO, A. et al. Composition and antimicrobial activity of essential oils from aromatic plants used in Brazil. Brazilian Journal of Microbiology, v.35, n.4, p.27580, 2004.

SCHWIERTZ, A. et al. In vitro activity of essential oils on microorganisms isolated from vaginal infections. International Journal of Aromatherapy, v.16, n.3-4, p.169-74, 2006.

SIKKEMA, J.; BONT, J.A.M.; POOLMAN, B. Mechanisms of membrane toxicity of hydrocarbons. Microbiological Reviews, v.59, n.2, p.201-22, 1995.

SIMÕES, C.M.O.; SPITZER, V. Óleos voláteis. In: SIMÕES, C.M.O. et al. Farmacognosia: da planta ao medicamento. Porto Alegre/Florianópolis: Editora da UFRGS/Editora da UFSC, 2003, p.468-95.

YURAYART, C. et al. Comparative analysis of the frequency, distribution and population sizes of yeasts associated with canine seborrheic dermatitis and healthy skin. Veterinary Microbiology, v.148, n.2-4, p.356-62, 2011. 